Military Technical College Kobry Elkobbah, Cairo, Egypt

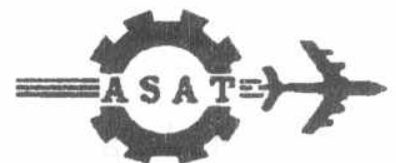

$8^{\text {th }}$ International Conference on Aerospace Sciences \& Aviation Technology

\title{
THE EFFECT OF THE EARTH'S GRAVITATIONAL FIELD ON PHOTONS WAVE LENGTHS OF UP- DOWN- LINK INFORMATION
}

M. MELEK ${ }^{1}$

\section{ABSTRACT}

It is pointed out that there exist real discrepancies between the used theories and experimental measurements in two phenomena on astronomi$\mathrm{cal}$ and laboratory scales. Those two phenomena are concerned with the motion of spinning particles in gravitational fields. A thurough study is presented on different theoretical possibilities of searching for a feasable solution for the existing puzzling discrepancies. One theoretical possibility; in which one may find an interpretation for these discrepancies; is exposed. This possibility is based upon taking into considerations, spin- gravity interaction when spinning particles are moving in gravitational fields. The obtained results; due to such possibility; are applied to calculate the effect of the Earth's gravitational field on photons wave lengths of up- down- link information.

\section{Key Words:}

Applied Gravitational Physics - Space Physics - Space Data Transmission - Space- Ground Communications.

\footnotetext{
${ }^{1}$ Associate Professor, Astronomy and Meteorology Department, Faculty of Science, Cairo University, Giza, Orman, Egypt. e.mail: melek@frcu.eun.eg
} 


\section{Introduction}

It is known that the transmission of data and informations from satellites to ground stations and viceversa, is carried by photons which are massless and spin-1 particles. The study of the effect of any gravitational field on the motion of photons is done usually by using the general relativistic equation of motion for photons, which is given by (cf.[1]):

$$
\frac{d^{2} u}{d \phi^{2}}+u=3 m u^{2}
$$

where $u=\frac{1}{r}, r, \phi$ are the radial and the third components of the spherical polar coordinate system $(r, \theta, \phi), m=\frac{G M}{c^{2}}, G$ is the gravitational constant, $M$ is the mass of the source of the gravitational field and $c$ is the velocity of light. It is clear from (1) that when there is no gravitational field (i.e. $m=0$ ), the light will move on a straight line given by the following differential equation:

$$
\frac{d^{2} u}{d \phi^{2}}+u=0
$$

Equation (1) is used to calculate the deflection of light in spherically symmetric gravitational field. The grvitational field does not change the direction of photons only, but also it changes their wave lengths while moving from their source to recievers. The known and confirmed result concerning the effect of spherically symmetric gravitational field on photons wave length, is given as follows [1]:

$$
\Delta \lambda=\lambda_{r}-\lambda_{s}=\frac{G M}{c^{2}}\left(\frac{1}{R_{s}}-\frac{1}{R_{r}}\right) \lambda_{s},
$$

where: $\lambda_{r}$ is the wave length at the reciever, $\lambda_{s}$ is the wave length at the source, $R_{r}$ and $R_{s}$ are the distances between the the center of the source of the gravitational field and the reciever and the source of photons respectively. It is clear from (3) that the wave length at 
the source equals to the wave length at the reciever when there is no gravitational field. The results (1) and (3) were obtained from the equations of motion of photons which are not capable to account for an interaction between the quantum spin of the moving photons and the background gravitational field.

This represents a long standing problem in gravitational physics in which physicists and astronomers are looking for an answer to the following question:

"How does a classical gravitational field affect the wave length of moving photons taking into considerations such suggested interaction?"

Two puzzling phenomena may provide reasonable motivation to search for such interaction. The first phenomenon is concerned with the real discrepancy between the used theory (Newtonian Gravity) and the measurements of the $\mathrm{COW}$-experiment. This experiment measures the phase difference between two beams of thermal Neutrons due to the effect of the Earth's gravitational field, provided that one of them is more closer to the Earth's surface than the other [2], [3], [4]. In the third version of this experiment [5], the measurements of the phase shift; due to the Earth's gravitational field; show that the experimental results are lower than the theoretical calculations; based on Newtonian mechanics; by about $8\left(10^{-3}\right)$. This discrepancy has no interpretation so far. There are two possibilities to find a feasable interpretation for such discrepancy; either $\mathbf{1}$. it is due to the experimental artifact, or $\mathbf{2}$. it is related to the basis of the used theory (Newtonian gravity). The first possibility was and is still explored by Layer and greene [6] and then again by Arife, Dewey, Greene, Jacobson and Werner [7]. The outcome of these investigations shows that the experimental results may be higher than the theoretical calculations by about $3 \cdot 7\left(10^{-3}\right)$. However, this study provides another discrepancy and it is not a conclusive one yet. Therefore, there is still a room to explore the second possibility. 
The second puzzling phenomenon is concerned with the arrival of the radiation (particles) produced by the explosion of the supernova SN1987A to the Earth's detectors (Kamiokande). Among astrophysicists it is accepted that Neutrinos; which are spin - $\frac{1}{2}$ and may be massless particles; were emitted from SN1987A couple of tens of hours before Photons, but they were recieved at the Earth's detectors three hours only before Photons [8], [9], [10], [11], [12]. Hence, the following question emerges:

"Why Neutrinos were delayed more than Photons while travelling through the gravitational field of the Milky Way galaxy, inspite of the accepted fact that both particles are treated as massless and are supposed to move along the same trajectory?"

Before any trial to answer the previous question, the following three questions should be answered:

1. What is the time difference between the emission of both particles?

2. Are Neutrinos and Photons moving; from their source to the Earth's detectors; along the same general relativistic trajectory for massless particles although one of them has spin $\frac{1}{2}$ while the other has spin 1 ?

3. Should Neutrinos be treated as massive particles?

In case that the answer of the first question is: "the time difference between their emission is approximately three hours", then chere is no puzzle at all. But in case that the answer is:" the time difference between their emission at the source and their arrival to the Earth's detectors is different", then this represents a real discrepancy between observations and theories determining the trajectories of massless spinning particles moving in a gravitational fields. Regarding the third equation, there are several authors are trying to investigate the possibility that Neutrinos possess an upper limit on 
their masses of order $(5 \mathrm{eV})$ for electron neutrino $\left(\nu_{e}\right),(160 \mathrm{KeV})$ for muon one $\left(\nu_{\mu}\right)$ and $(24 \mathrm{MeV})$ for tau one $\left(\nu_{\tau}\right)$ respectively (cf. [13]). Therefore, one can use the delay time of the Neutrinos to put limits on their masses which should be consistent with those limits comming from quantum field theory considerations. This possibility may present solution to the puzzle of Neutrinos delay comming from SN1987A without referring that to spin - gravity interaction. But still remains the discrepancy in the COW - experiment which may be referred to spin gravity interaction.

However, the previous two puzzling phenomena suffered from real discrepancies between the used theories; either Newtonian or Einesteinian (general relativistic); and the experimental measurments. Theories used in both phenomena do not account for an interaction between the particle's quantum spin and the background gravitational field. It is worth to point out here that one of those phenomena is taking place on the laboratory scale, where Newtonian and non-relativistic quantum mechanics equations of motion are used. While the second one is occuring on the astronomical scale, where the general relativistic equations of motion for massless particles are used.

The author does think that those two phenomena are providing us with two evidencies, on different scales, about. such interaction which may exist in nature and gives us a feasable solution to the existing puzzling discrepancies.

In this article a thurough study is presented on different theoretical possibilities of searching for a feasable solution to the existing discrepancies. One theoretical possibility, in which one may find an interpretation for these discrepancies, is exposed. This possibility is based upon taking into considerations, spin - gravity interaction when spinning particles are moving in a gravitational field. The obtained results, due to such possibility, are applied to calculate the effect of the Earth's gravitational field on photons wave lengths of up- down- link information. 


\section{THEORETICAL TRIALS TO FIND SPIN - GRAVITY INTERACTION:}

It is a long standing problem in gravitational physics to find out how the quantum spin of a particle interacts with a classical (non-quantized) gravitational field. There are two theoretically equivalent standard approaches for tackling this problem. One of them is to formulate the quantum theory for spinning particle in a background gravitational field. This is done by adding the pure gravity Hilbert action to the action of free spinor field; for spin $\frac{1}{2}$ particles; after writting it in a covariant form. This approach produces the general relativistic Dirac equation besides equations relating the space-time curvature to the energy-momentum tensor andthe space-time torsion to the spin density tensor (cf. [14], [15], [16], [17] and references therein). The general relativistic Dirac equation is given by:

$$
\left(\gamma^{\mu} \mathbf{D}_{\mu}-m\right) \Delta_{D F}\left(x_{N}, x_{0}\right)=\frac{2 \imath \hbar}{(-g)^{\frac{1}{2}}} \delta\left(x_{N}-x_{0}\right),
$$

where

$$
\mathbf{D}_{\mu}=\imath \hbar D_{\mu}=\imath \hbar\left(\mathrm{D}_{\mu}+\frac{1}{2} \gamma_{\mathrm{jk} \mu} \sigma^{\mathrm{jk}}\right)
$$

$\mathrm{D}_{\mu}$ is the Riemanian covariant derivative with respect to $x^{\mu}, \gamma_{j k \mu}$ are the Ricci's coefficients of rotation which are given in terms of vierbein vectors $\lambda_{j \nu}$ as follows:

$$
\gamma_{j k \mu}=-\left(\mathrm{D}_{\mu} \lambda_{j \nu}\right) \lambda_{k}{ }^{\nu},
$$


$\lambda_{k \mu}$ are the four vectors field (vierbein) which are erected at each point in space-time,

$$
\begin{gathered}
\sigma^{j k}=\left(\begin{array}{llll}
0 & \alpha_{x} & \alpha_{y} & \alpha_{z} \\
-\alpha_{x} & 0 & -\imath \Sigma_{z} & \imath \Sigma_{y} \\
-\alpha_{y} & \imath \Sigma_{z} & 0 & -\imath \Sigma_{x} \\
-\alpha_{z} & -\imath \Sigma_{y} & \imath \Sigma_{x} & 0
\end{array}\right)=\frac{1}{4}\left(\gamma^{j} \gamma^{k}-\gamma^{k} \gamma^{j}\right), \\
\alpha_{l}=\left(\begin{array}{ll}
\sigma_{l} & 0 \\
0 & -\sigma_{l}
\end{array}\right), \Sigma_{l}=\left(\begin{array}{ll}
\sigma_{l} & 0 \\
0 & \sigma_{l}
\end{array}\right), \gamma^{0}=\left(\begin{array}{ll}
0 & 1 \\
1 & 0
\end{array}\right), \gamma^{l}=\left(\begin{array}{ll}
0 & -\sigma_{l} \\
\sigma_{l} & 0
\end{array}\right),
\end{gathered}
$$

$l=1,2,3, j, k, \mu, \nu=0,1,2,3, \sigma_{l}$ are the Pauli spin matricies, $\Delta_{D F}$ is the Dirac- Feynman propagator, $g$ is the determinant of the metric tensor representing the gravitational field, $m$ is the mass of the moving particle, and $\delta\left(x_{N}-x_{0}\right)$ is the Dirac delta function.

It is clear from (5) that the potential of such interaction between the quantum spin of a spin - $\frac{1}{2}$ particle and the background gravitational field is expressed by the presence of the term $\frac{1}{2} \gamma_{j k \mu} \sigma^{j k}$ in the general relativistic Dirac equation. The matrix $\sigma^{j k}$ in this potential characterizing the spin of the particle as a pure quantum mechanical observable. But the Ricci's coefficients of rotation are characterizing the background gravitational field. This means that the spin of a particle may interact with the gravitational field via the Ricci's coefficients of rotation.

The second approach is to describe classically the spin degrees of freedom for a moving particle and then quantize its classical motion to get-a consistent theory, which includes such interaction between the quantum spin of a particle and the background gravitational field. This approach gives the following classical equations of motion for spin - $\frac{1}{2}$ particle moving in a gravitational field (cf. [18], [19], [20] [21], [22]).

$$
\frac{d^{2} x^{\mu}}{d S^{2}}+\left\{\begin{array}{c}
\mu \\
\rho \nu
\end{array}\right\} \frac{d x^{\rho}}{d S} \frac{d x^{\nu}}{d S}=\frac{1}{2 m} R_{i j \rho}^{\mu} S^{i j} \frac{d x^{\rho}}{d S},
$$


where

$$
\left\{\begin{array}{c}
\mu \\
\rho \nu
\end{array}\right\}=\frac{1}{2} g^{\mu \sigma}\left(g_{\rho \sigma, \nu}+g_{\sigma \nu, \rho}-g_{\rho \nu, \sigma}\right)
$$

are the Christoffel symbols, $g_{\mu \nu}$ is the metric tensor representing the background gravitational field,

$$
\begin{gathered}
R_{i j \rho \nu}=\lambda_{i}^{\sigma} \lambda_{j}^{\mu} R_{\sigma \mu \rho \nu}, \\
R_{i j \rho \nu}=\gamma_{i j \nu, \rho}-\gamma_{i j \rho, \nu}+\gamma_{j \nu}^{k} \gamma_{i k \rho}-\gamma_{j \rho}^{k} \gamma_{i k \nu},
\end{gathered}
$$

$S^{i j}$ is the spin tensor of the particle, and $m$ is the mass of the moving spinning particle.

It is clear from the right hand side of (9) that the force $F^{\mu}$. acting on a moving massive spinning particle due to the interaction between the spin of the particle and the background gravitational field is given by:

$$
F^{\mu}=\frac{1}{2 m} R_{i j \rho}{ }^{\mu} S^{i j} \frac{d x^{\rho}}{d S},
$$

where $R_{i j \rho}{ }^{\mu}$ is characterizing the background gravitational field, and $m$, $S^{i j}$ and $\frac{d x^{\rho}}{d S}$ are characterizing the moving particle. Therefore, the curvature $R_{i j \rho}{ }^{\mu}$, which is calculated from the Ricci's coefficients of rotation, is the propert.y of the background gravitational field participating in such interaction. Therefore, from (4) and (9) one may conclude that the Ricci's coefficients of rotation is the intrinsic property of the background gravitiational field which interacts with the spin degrees of freedom of spim, - $\frac{1}{2}$ particles.

Recently, Wanas, Melek and Kahil [23] have developed an approach in which the deviation from the geodesic motion; described in a wider geometry than the Riemannian one; may be considered as a window to express interaction between the spin of a moving particle and a classical background gravitational field. As it is known, Riemannian geometry posseses only two equations of motions: 1 . geodesics for massive objects or particles, 2. null geodesics for massless particles. Both equations are not capable to account for spin - gravity interactions. 
Einestein [24], Mikhail, Wanas [25], Moller [26], and Hayashi, Shirafuji [27] have used wider geometry than the Riemannian one to account for other types of classical interactions. This geometry is the absolute parallelism (AP) geometry. In this geometry, one can define three new different covariant derivatives related to three different affine connections (cf. [28] and references therein). Bazanski [29] has developed an approach to obtain both geodesic and geodesic deviation equations, in Riemannian geometry, using the following Lagragian:

$$
L=g_{\mu \nu} U^{\mu} \frac{D \psi^{\nu}}{D S}
$$

where: $U^{\mu}=\frac{\partial x^{\mu}}{\partial S}, \psi^{\nu}=\frac{\partial x^{\nu}}{\partial \tau}$ is the deviation vector, $S, \tau$ are evolution parameters and

$$
\frac{D \psi^{\nu}}{D S}=\frac{\partial \psi^{\nu}}{\partial S}+\left\{\begin{array}{l}
\nu \\
\rho \sigma
\end{array}\right\} \psi^{\rho} U^{\sigma}
$$

Wanas et. al. [23], have generalized Bazanski Lagrangian using the three different affine connections in AP-geometry and have obtained the following three equations respectively:

$$
\begin{gathered}
\frac{d L^{-\mu}}{d S_{-}}+\left\{{ }_{\alpha \beta}^{\mu}\right\} U^{\alpha} U^{\beta}=0, \\
\frac{d U^{\mu}}{d S_{0}}+\left\{{ }_{\alpha, 3}^{\mu}\right\} U^{\alpha} U^{\beta}=-\frac{1}{2} \Lambda_{(\alpha \beta)}{ }^{\mu} U^{\alpha} U^{\beta}, \\
\frac{d U^{\mu}}{d S_{+}}+\left\{{ }_{\alpha \beta}^{\mu}\right\} U^{\alpha} U^{\beta}=-\Lambda_{(\alpha \beta)}{ }^{\mu} U^{\alpha} U^{-\beta},
\end{gathered}
$$

where $S_{-}, S_{0}, S_{+}$are the evolution parameters along the paths,

$$
\Lambda_{\mu \nu}^{\rho}=\Gamma_{\mu \nu}^{\rho}-\Gamma_{\nu \mu}^{\rho},
$$

is the torsion tensor field, $\Gamma_{;, L \nu}^{\rho}$ is a non-symmetric $\mathrm{GL}(4)$ connection in AP-geometry, and $\Lambda_{(\alpha \beta)}{ }^{\mu}$ is the symmetric part of the torsion in $\alpha$ and $\beta$. 
A striking feature of the three equations (15), (16), (17) is the jump of step $\frac{1}{2}$ among them. Wanas [30] has constructed an AP-geometry with a generalized affine connection to get a family of paths in which the coefficient of the torsion term jumps with a step of one- half. This family of paths is given as follows:

$$
\frac{d U^{\mu}}{d \tau}+\left\{{ }_{\alpha \beta}^{\mu}\right\} U^{\alpha} U^{\beta}=-\frac{n}{2} \alpha \Lambda_{(\alpha \beta)}{ }^{\mu} U^{\alpha} U^{\beta},
$$

where $\alpha$ is the fine structure constant $\left(\alpha=\frac{1}{137}\right)$ and $n=0,1,2, \ldots$ is a natural number which will be 0 for spinless particles, 1 for spin- $\frac{1}{2}$ particles, 2 for spin- 1 particles... and so on.

Wanas, Melek and Kahil [31] have used this equation, successfully, to find out a possible and satisfactory interpretation of the discrepancy in the COW- experiment as well as to find a reasonable explanation of the delay of Neutrinos more than Photons comming from SN1987A.

\section{The Effect of Spin - Gravity Interaction on the Wave Lengths of Photons Moving in the Earth's Gravitational Field:}

Now, let us suppose that a satellite moving on a distance $1000 \mathrm{~km}=$ $10^{8} \mathrm{~cm}$ above the Earth,s surface. Therefore Photons with wave length $\lambda_{s}$ carrying informations from the satellite to a ground station on the Earth's surface, will be recieved with a wave length $\lambda_{r}$ which is given by:

$$
\lambda_{r}=\lambda_{s}+\frac{G M_{\oplus}}{c^{2}}\left(\frac{1}{R_{s}}-\frac{1}{R_{r}}\right) \lambda_{s},
$$

where: $G=6.673\left(10^{-8}\right) \mathrm{cm}^{3} \mathrm{gm}^{-1} \mathrm{sec}^{-2}, c=2.998\left(10^{10}\right) \mathrm{cmsec}^{-1}, M_{\oplus}=$ $(5.977 \pm 0.004)\left(10^{27}\right) \mathrm{gm}$ is the Earth's mass, $R_{\oplus}=6.371\left(10^{8}\right) \mathrm{cm}$ is the Earth's mean radius, $R_{s}=R_{\oplus}+10^{8} \mathrm{~cm}$, and $R_{r}=R_{\oplus}$. Hence,

$$
\lambda_{r}=\lambda_{s}-0.9\left(10^{-10}\right) \lambda_{s} \text {. }
$$


In the case that the source of photons is a ground station, on the Earth's surface, and the reciever is in the satellite, one obtains:

$$
\lambda_{r}=\lambda_{s}+0.9\left(10^{-10}\right) \lambda_{s} .
$$

The results (21) and (22) are obtained from using (20) which is obtained from the equations of motion of photons in gravitational field without taking into considerations the photon's spin - Earth's gravity interaction.

Since the Earth's gravitational field can be considered as a spherically symmetric, weak and static field (i.e. $g_{\mu \nu}=\eta_{\mu \nu}+\epsilon h_{\mu \nu}, \epsilon^{2} \simeq 0, h_{\mu \nu, 0}=$ 0 ), then the linearized form of equation (19) gives the following equation

$$
\frac{d^{2} x^{i}}{d t^{2}}=-\left(1-\frac{n}{2} \alpha\right) \frac{\partial \phi_{N}}{\partial x^{i}}
$$

where: $i=1,2,3$ and $\phi_{N}$ is the Newtonian potential of the Earth which is defined as follows:

$$
\phi_{.:}=\frac{c^{2}}{2}\left(1-g_{00}\right)
$$

Therefore, massless spin - 1 particle (photon) feels the following potential

$$
\varphi_{s g}=(1-\alpha) \phi_{N}
$$

where $\phi_{s g}$ stands for the potential of the gravitational field, when spin - gravity interaction is taken into considerations. Hence the equations (3) and (20) will be modified; due to such interaction, to following expression:

$$
\lambda_{r}=\lambda_{s}+\frac{G \cdot M_{\oplus}}{c^{2}}(1-\alpha)\left(\frac{1}{R_{s}}-\frac{1}{R_{r}}\right) \lambda_{s}
$$

Using such expression for photons emitted from a satellite at distance $1000 \mathrm{~km}$ from the Earth's surface, one obtains the following expression for the recieved wave length at the ground station:

$$
\lambda_{r}=\lambda_{s}-0.9\left(10^{-10}\right) \lambda_{s}+0.648\left(10^{-12}\right) \lambda_{s} .
$$


But in the case that the source of photons is a ground station and the reciever is in the satellite, the recieved wave length will read:

$$
\lambda_{r}=\lambda_{s}+0.9\left(10^{-10}\right) \lambda_{s}-0.648\left(10^{-12}\right) \lambda_{s} .
$$

It is clear from the third term in (27) and (28) that it gives the effect of spin - Earth's gravity interaction on the wave length of photons carrying data and informations from a satellite to ground stations and viceversa.

\section{Epilogue}

It is clear from the history of the different trials to find spin - gravity interaction that these trials were dealing only with spin- $\frac{1}{2}$ particles case and not with other particles with spin different from $\frac{1}{2}$. This is due to the following two reasons:

1. the quantum theory for spin $\frac{1}{2}$ particles (Dirac theory) is well established and successfully formulated in the presence of gravitational field,

2 . there are several successful classical models for spin- $\frac{1}{2}$ particles which is formulated in the presence of gravitational field.

However, the recent suggested approach gives an equation of motion of any particle with any spin according to the value of $n$. According to equation (19), it is clear that this new suggested spin - grávitȳ' interaction is spin orientation independent, i.e. the gravitational field is not able to distinguish between different spin polarization states of different spinning particles (e.g. spin up or spin down for spin- $\frac{1}{2}$ particles). This phenomenon is similar to the known phenomenon in which gravitational fields are not able to distinguish between positive or negative charges.

Also, it is clear from equation (25) that this suggested interaction by this new approach is not changing the type of dependence of the gravitational potential on the independent variables but it is a rescaling of the known classical potetial by the fine structure constant. 
Finally, it is clear from (27) and (28) that the fluctuations in the wave lengths of photons; due to the Earth's gravitational field and considering spin - gravity interaction; carrying data and informations from a 1000 $\mathrm{km}$. distant satellite from the Earth's surface to ground stations and viceversa, is given by:

$$
\frac{\Delta \lambda}{\lambda_{s}}=-0.9\left(10^{-10}\right)\left(1-0.72\left(10^{-2}\right)\right)
$$

in case that the source of informations is the satellite, and

$$
\frac{\Delta \lambda}{\lambda_{s}}=0.9\left(10^{-10}\right)\left(1-0.72\left(10^{-2}\right)\right),
$$

in case that the source of informations is the ground station.

\section{References}

[1] Misner C. W., Thorne K. S. and Wheeler J. A., "Gravitation", Freeman, San Fransisco, 1973.

[2] Overhauser A. W. and Colella R., Phys. Rev. Lett. vol.33, No. 20, 1237, 1974.

[3] Colella R., Overhauser A. W. and Werner S. A., Phys. Rev. Lett. vol. 34, No. 23, 1472, 1975.

[4] Staudenmann J. L., Werner S. A., Colella R. and Overhauser A. W. ,Phys. Rev. A, vol. 21, No. 5, 1419, 1980.

[5] Werner S. A., Kaiser H., Arif M. and Clother R., Physica B, vol. 151, No. 22, 1988.

[6] Layer H. P. and Greene G. L., Phys. Lett. A, 155, 450, 1991.

[7] Arif M., Dewey M. S., Greene G. L., Jacobson D. and Werner S. A., Phys. Lett. A, 185, 154, 1994. 
[8] Longo M. J., Phys. Rev. D, 3276, 1987.

[9] Longo M. J., Phys. rev. Lett., 60, 173, 1988.

[10] De rujula A., Phys. Lett., 193, 514, 1987.

[11] Krauss L. M. and Tremaine S., Phys. Rev. Lett.,60, 176, 1988.

[12] Schram D. N. and Truran J. W., Phys. Rep., 189, 89, 1990.

[13] Pagel B. E. J., " Nucleosynthesis and Chemical Evolution of Galaxies", P. 39, Cambridge University Press, 1997.

[14] Utiyama R., Phys. Rev. 101, 1597, 1956.

[15] Kibble T. W., J. Math. Phys. 2, 212, 1961.

[16] Nieh H. T. and Yan M. L., Ann. Phys. 138, 237, 1982.

[17] Yong-Shi W. and Zee A., J. Math. Phys. 25, 9, 1984.

[18] Papapetrou A., Proc. Roy. Soc. A 209, 248, 1951.

[19] Barducci A., Casalbouni R. and Lusana L., Nucl. Phys. B124, 521, 1977.

[20] Galvao C. and Teitelboim C., J. Math. Phys. 21, 1863, 1980.

[21] Balek V., Melek M. and Petras M., Czech. J. Phys. B37, 1321, 1987.

[22] Melek M., acta Phys. Slov. 38, 3, 146, 1988.

[23] Einestein A., Sitz. Preuss Akad. Wiss. 1, 1, 1929.

[24] Mikhail F. I. and Wanas M. I., Proc. Roy. Soc. London, A 356, 471, 1977.

[25] Moller C., Mat. Fys. Medd. Dan. Vid. Selek, A 39, 1, 1978. 